\title{
Estrategias de aprendizaje autónomo en la comprensión lectora de estudiantes de secundaria
}

\section{Autonomous Learning Strategies in the Reading Comprehension of High School Students}

\author{
Daniela Medina Coronado* \\ Universidad César Vallejo, Lima, Perú \\ ORCID: https://orcid.org/0000-0002-9180-7613 \\ Mercedes María Nagamine Miyashiro \\ Universidad César Vallejo, Lima, Perú \\ ORCID: https://orcid.org/0000-0003-2763-740X
}

Recibido 01-09-18 Revisado 20-09-18 Aprobado 20-12-18 En línea 04-01-19

*Correspondencia

Email: danmedina27@hotmail.com

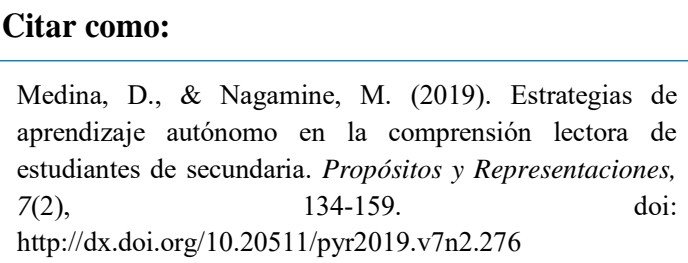

\section{Citar como:}

\& Nagamine, M. (2019). Estrategias de estudiantes de secundaria. Propósitos y Representaciones,

http://dx.doi.org/10.20511/pyr2019.v7n2.276 


\section{Resumen}

Al observar en las aulas de clase del nivel secundaria, así como del nivel superior la forma como los adolescentes y jóvenes estudian surgió la idea de conocer si cuentan con estrategias para el aprendizaje independiente y de si esta variable explicaba en alguna medida un problema crítico en el Perú: la comprensión lectora. Es por ello, que se planteó como propósito del estudio, conocer si las estrategias de aprendizaje autónomo, sus dimensiones e indicadores tienen algún poder explicativo sobre la comprensión lectora. Bajo un diseño correlacional causal de carácter exploratorio, se aplicaron los siguientes instrumentos: Cuestionario de estrategias de trabajo autónomo (CETA) que evalúa las estrategias de ampliación, colaboración, conceptualización, planificación, preparación de exámenes y participación y prueba PECL 2 para medir niveles de comprensión de la lectura: literal, inferencial y criterial en un grupo de 144 estudiantes de IE de los distritos de Majes en Arequipa y Villa Rica en Pasco. Entre los resultados más importantes se obtuvo que el $79.33 \%$ de estudiantes alcanzaron un nivel en proceso en cuanto al uso de estrategias de aprendizaje autónomo y el 62.7\% alcanzó el mismo nivel en la comprensión lectora; así mismo, se halló que el $34.4 \%$ de la variable comprensión lectora es explicada por el uso de estrategias de aprendizaje autónomo, de ese total, un $25.8 \%$ por las estrategias de ampliación, $14.3 \%$ por las estrategias de colaboración, $28.7 \%$ por las estrategias de conceptualización, $19.6 \%$ por las estrategias de preparación de exámenes y finalmente un $19.5 \%$ por las estrategias de participación.

Palabras clave: Estrategias de aprendizaje autónomo; Comprensión lectora; Estudiantes de secundaria.

\section{Summary}

When observing in the high school as well as higher education classrooms, the way adolescents and young people study, the idea of knowing if they have strategies for autonomous learning and if this variable explained to some extent a critical problem in Peru arose: reading comprehension. That is why it was proposed as a purpose of the study, to know if the autonomous learning strategies, its dimensions and indicators have some explanatory power on reading comprehension. Under a causal correlational design of an exploratory nature, the following instruments were applied: Autonomous work strategies questionnaire (CETA) that evaluates the strategies of expansion, collaboration, conceptualization, planning, preparation of exams and participation and PECL 2 test to measure levels of reading comprehension: literal, inferential and evaluative in a group of 144 students from educational institutions from the districts of Majes in Arequipa and Villa Rica in Pasco. Among the most important results was that $79.33 \%$ of students reached a level in process regarding the use of autonomous learning strategies and $62.7 \%$ reached the same level in reading comprehension. Likewise, it was found that $34.4 \%$ of the reading comprehension variable is accounted for the use of autonomous learning strategies. Out of that total, $25.8 \%$ for expansion strategies, $14.3 \%$ for collaboration strategies, $28.7 \%$ for conceptualization strategies, $19.6 \%$ for exam preparation strategies and finally, $19.5 \%$ for participation strategies.

Keywords: Autonomous Learning Strategies; Reading Comprehension; High School Students. 


\section{Introducción}

¿Han desarrollado los estudiantes de la educación básica regular las capacidades mínimas necesarias para un aprendizaje autónomo que les permita enfrentar los retos de la educación superior peruana, de la globalización de la información o de la transformación digital de la educación? ¿Al término de la educación básica regular, comprenden los estudiantes, realmente lo que leen? Todas estas interrogantes surgen porque la realidad revela que los estudiantes presentan una serie de deficiencias y carencias en comparación a los estándares mínimos exigibles.

Durante la Colonia hasta el siglo XX, la educación en el Perú ha sido impartida bajo el modelo conductista, en el que se brindaban conocimientos de manera memorística sin mayor participación crítica y reflexiva del estudiante, entrado el siglo XXI el modelo educativo ha sido el constructivista, aún no se tiene un pleno dominio por parte de las instituciones educativas, del docente y del propio estudiante de los nuevos procesos de enseñanza - aprendizaje. Ello ha generado que muchas competencias básicas y hábitos de estudio, sigan sin lograrse en los estudiantes, ya que persiste la pasividad para el autoaprendizaje aun aplicándose estrategias constructivistas, ello se evidencia porque son poquísimos los estudiantes que llegan a las aulas con material previamente revisado, es decir con pobres hábitos para el aprendizaje autónomo.

Esta problemática se observa con mayor incidencia en las provincias del Perú, por sus mayores niveles de pobreza y deficiente infraestructura, donde aún existen escuelas polidocentes, sin los recursos y materiales educativos mínimos para asegurar una enseñanza de calidad, y menos aún, acorde a los cambios vertiginosos que se están presentando con la educación 3.0, debido a que la brecha digital actual es muy grande.

Para que estudiante logre un aprendizaje autónomo, sobre todo en educación superior, debe haber desarrollado un pensamiento consciente, reflexivo, crítico, que sea capaz de utilizar estrategias para aprender por sí mismo de manera independiente. Para ello, es necesario que el estudiante sea consciente de sus capacidades, habilidades y actitudes hacia el aprendizaje: iniciativa para ser proactivo, para adquirir más conocimientos que los proporcionados por el docente; responsabilidad, capacidad de colaboración con el docente y con sus pares con el fin de que se pueda trabajar en equipo con un nivel de comunicación adecuado para transmitir ideas en el marco de tolerancia; habilidad para resolver problemas, estar preparado de antemano para las evaluaciones; autodisciplina para planificar su propio aprendizaje; y finalmente, persistencia para no desertar.

Por lo expuesto, se puede inferir que el nivel promedio de aprendizaje autónomo no es el deseado, por la observación realizada al estudiante, su desempeño en el aula, la calidad de trabajos que presenta; si no es correctamente asesorado y acompañado por el docente. El estudiante no ha desarrollado el hábito de utilizar estrategias de aprendizaje de manera sistemática que le permita el logro de mejores resultados académicos. De acuerdo con López (2002), quien corrobora que los expertos afirman que hay una necesidad imperiosa de enseñar a aprender, debido a que la falta de uso de estrategias de aprendizaje por parte de los estudiantes incrementa el fracaso escolar, además se evidencia la escasa capacidad de los estudiantes de interpretar y transferir información, habilidades que están vinculadas con las competencias lectoras.

En cuanto a la comprensión lectora, (Gaines \& Meca 2013 citado por Ceplan 2014) menciona que los países que están liderando los rankings del sector educativo como Corea del 
Sur, Singapur y Finlandia, están enfatizando el desarrollo integral de los ciudadanos; es decir, además de generar competencias en matemáticas y comprensión lectora, están desarrollando habilidades blandas en espacios participativos, colaborativos y de tolerancia, con dominio de las tecnologías de la información. Los cuestionamientos realizados al inicio facilitan dar una mirada a lo que está sucediendo en el país y realizar un diagnóstico que permita tener una línea base de partida para proponer un redireccionamiento para del proceso de enseñanza - aprendizaje en función de los cambios que se están dando en el ámbito educacional a nivel mundial.

En el Perú, de acuerdo al consenso llegado por las instituciones involucradas en la educación, es que el cuarto objetivo para una educación de calidad es la de garantizar una educación inclusiva, equitativa y de calidad y promover oportunidades de aprendizaje durante toda la vida para todos (Ceplan, 2017), de acuerdo a la evaluación realizada en el nivel de comprensión lectora en estudiantes de segundo grado de primaria, se observa que se ha incrementado de $15,9 \%$ en el año 2007 al 49,8\% en el año 2015, lo cierto es que frente a esta realidad nacional de niveles de comprensión lectora tan bajos, el cuestionamiento es si los estudiantes logran aprender y desarrollar las competencias que propone el Ministerio de Educación.

Para dar respuesta a este cuestionamiento, de acuerdo a El Comercio (2016), la prueba PISA 2015 determina que el Perú, si bien es el país de Latinoamérica que ha mejorado más a nivel general, aún se encuentra en el puesto 62 de 70 participantes por debajo de Colombia, Chile, Costa Rica y Uruguay. Los resultados obtenidos están por debajo del promedio mundial de la OCDE; los países asiáticos como Singapur, China y Corea del Sur lideran los resultados PISA.

Las causas por las existen bajos niveles de comprensión lectora son diversas: falta de motivación, pobreza de vocabulario, desarrollo de habilidades cognitivas involucradas, escasos conocimientos previos, desconocimiento y/o falta de dominio de estrategias de comprensión y de estrategias metacognitivas por mencionar algunas (López, 2010). Bajo el concepto que en la sociedad actual es necesario el hecho de que los estudiantes sean capaces de aprender por sí mismos, comprendiendo lo que leen, el primer paso es conocer cuál de las variables anteriormente mencionadas explica de manera importante a la comprensión lectora; por ello el propósito del presente estudio fue conocer en qué medida las estrategias de aprendizaje autónomo, inciden en la comprensión lectora.

Entre los estudios vinculados con el presente se encuentran los de Koosha, Abdollahi y Karimi (2016), quienes investigaron en la Universidad Islámica Azad sobre la relación existente entre tres variables autoestima, la autonomía y la comprensión de lectura, en una muestra aleatoria de 121 estudiantes de lengua extranjera, hallando una correlación alta de 0.911 entre el aprendizaje autónomo y comprensión lectora y un R2 de 0.82 al 95\% de nivel de confianza. En la misma universidad Zarei y Gahremani se propusieron seis años antes investigar sobre la relación entre aprendizaje autónomo y comprensión lectora, en una muestra de 68 estudiantes de cuatro universidades de Irán, los resultados obtenidos a través del Coeficiente de correlación de Pearson y de regresión ANOVA de una vía, demostraron una relación significativa pero baja $\mathrm{r}=0.264 \mathrm{a}$ un nivel de confianza del 95\%, caso contrario los resultados del análisis de regresión para identificar cuál de las dimensiones de la escala de Aprendizaje autónomo predice mejor la comprensión lectora no fueron estadísticamente significativos. De la misma manera, Gaeta (2015) presentó los resultados de su investigación: aspectos personales que favorecen la autorregulación del aprendizaje en la comprensión de textos académicos en estudiantes universitarios de Valencia 
España, con el objetivo de realizar un análisis de las variables propuestas, trabajó con una importante muestra de 364 estudiantes de primer ciclo de universidad, los resultados del análisis de regresión demostraron que las metas que se plantea el estudiante interviene favorablemente en el uso de estrategias de autorregulación de su aprendizaje en la comprensión lectora.

Entre los estudios nacionales se encuentran la propuesta de Sevillano (2015) quien investigó la relación entre las variables: estrategias de aprendizaje, la motivación y la comprensión lectora, en 217 estudiantes de cuarto año de secundaria de básica regular, a los que se les aplicó el cuestionario multifactorial de estrategias de aprendizaje y motivación y la prueba DET para comprensión lectora respectivamente, comprobándose relación entre las variables estudiadas. Así mismo, una de las dimensiones del aprendizaje autónomo son las estrategias de colaboración, al respecto Guevara (2014) realizó un estudio con el objetivo de demostrar la existencia de la relación entre las Estrategias de aprendizaje cooperativo y la comprensión lectora, en una muestra de 85 estudiantes de la Facultad de Educación de la UNA de Madre de Dios aplicando dos cuestionarios de 40 ítems altamente confiables 0.882 y 0.839 , respectivamente. Los resultados obtenidos determinaron que no existe relación significativa entre las variables de estudio, tampoco entre las dimensiones estudiadas.

Es en este marco que se planteó como objetivo determinar la estrategia de aprendizaje autónomo predominante que incide en la comprensión lectora de los estudiantes del 5 to grado de secundaria de IE públicas de Pasco y Arequipa, partiendo de la pregunta ¿Cuál es la estrategia de aprendizaje autónomo predominante que incide en la comprensión lectora de los estudiantes del 5to grado de secundaria de IE públicas de Pasco y Arequipa??

\section{Estrategias de aprendizaje autónomo.}

Esteban, Ruiz y Cerezo (1996), consideran que las estrategias son las acciones que han sido organizadas de manera consciente, así como los procedimientos que parten de la iniciativa del propio estudiante, organizadas y planificadas por el propio aprendiz para resolver tareas concretas de aprendizaje. Estas estrategias de aprendizaje están ligadas a la metacognición, comportamiento estratégico incluye la deliberación y flexibilidad en la selección de los propios recursos y capacidades, planificación y evaluación de acciones o procedimientos.

En el orden de las ideas anteriores, las estrategias vienen a ser procesos que implica la toma de decisiones y el dominio procedimental sobre los pasos más apropiados que el estudiante deberá escoger para enfrentarse a una tarea (Mayor, Suengas \& González, 1993; Monereo, 1994). Las mismas que dependen del contexto del estudiante, así como las características de la situación concreta de aprendizaje teniendo como objetivo el logro del aprendizaje y de esta manera lograr la adquisición de competencias.

El uso adecuado de estas estrategias permitirá al estudiante desarrollar un aprendizaje autónomo, el estudiante tendrá el control y autorregulación de su proceso de aprendizaje, por lo tanto; conllevará al logro de las competencias. Es para ello fundamental que el estudiante adquiera un amplio repertorio de estrategias las mismas que serán escogidas de acuerdo a las características propias de cada estudiante. El desarrollo y aplicación de las estrategias de aprendizaje autónomo requiere en un primer momento del acompañamiento docente como un ente motivador y facilitador (López-Aguado, 2010). Para este estudio han sido consideradas las siguientes estrategias, propuestas por el mismo autor: 
Estrategias de ampliación, orientadas al desarrollo de las competencias informacionales de los estudiantes expresados en la capacidad que tiene el estudiante de búsqueda de información para ampliar la información que recibe el estudiante en aula, están referidas a consultar otras fuentes bibliográficas físicas o virtuales - uso de internet, así como realizar actividades complementarias, todo ello con el propósito de ampliar y mejorar la comprensión de algún concepto.

Estrategias de colaboración, referidas a conocer y utilizar todos los recursos que proporciona la institución educativa, realizar trabajos colaborativos con los compañeros de clase, intercambiando resúmenes, materiales, información de internet para resolver trabajos; así también disipar dudas con los compañeros de clase.

Estrategias de conceptualización, relacionadas con el uso de distintos organizadores de conocimientos como: esquemas, mapas mentales, mapas conceptuales, cuadros sinópticos, cuadros comparativos, entre otros, así como el uso de estrategias de lecturas como: el subrayado, sumillado, etc. para realizar resúmenes, son estrategias de conceptualización que van a desarrollar el aprendizaje autónomo.

Estrategias de preparación, el desarrollo y logro de un aprendizaje autónomo se dará a través de que el estudiante sea actor principal de su aprendizaje y ello empieza por planificar sus actividades académicas y el tiempo que le va a dedicar al estudio y a la realización de trabajos académicos y termina con la autoevaluación de su proceso de aprendizaje.

Estrategias de participación, el estudiante que aprende de manera autónoma es consciente de tiene que participar activamente en el proceso de aprendizaje desde la lectura de todo el material que le proporcione el docente, participar en las actividades organizadas en el aula como exposiciones, debates, prepararse para los exámenes entre otras actividades.

Estrategias de planificación, relacionadas con la actitud y las acciones que realiza el estudiante en aula como: participar activamente en aula, el tomar apuntes de la cátedra de los docentes así como el aporte u opiniones de los compañeros de clase, anotar las dudas propias para luego disiparlas con las estrategias de ampliación. Finalmente, estas estrategias buscan que el estudiante contraste los conocimientos que va adquiriendo.

\section{Comprensión lectora.}

La definición de comprensión lectora que fundamenta Solé (2007) es el entendimiento del lenguaje escrito por parte del lector, para ello participan: el texto, el lector y sus conocimientos previos, bagaje cultural suficiente, es necesario también que el lector posea habilidades que le permitan decodificar un texto, aporte con sus ideas, objetivos así como sus experiencias previas y finalmente tenga la capacidad de comprobar y rechazar pronósticos.

A la definición mencionada se puede contribuir la propuesta de Pinzás (2006) que para que se dé la comprensión lectora es necesario la interpretación de significados que le dé sentido al texto, para ello se debe desarrollar esta capacidad en el aprendiz de temprana edad, a través de la estimulación desde la interpretación de imágenes hasta la realización de preguntas para lograr la 
meta de comprender, se puede agregar que todo este proceso se da en un contexto socio cultural (Solórzano \& Montero, 2011).

Para que el estudiante logre comprender lo que lee, es importante la labor del docente, en el acompañamiento para el desarrollo y logro de esta competencia, como manifiesta Solé (2004) se debe tener objetivos claros a la hora de leer, saber que se espera de la lectura, interrogarse sobre la lectura y tener consciencia de si se está comprendiendo, realizar inferencias, imaginar. Así también el docente debe enseñar a que el estudiante diferencie las ideas principales de las secundarias, a realizar resúmenes, esquemas. Por lo que se puede concluir que para comprender una lectura no basta con leer un texto sino acompañar a la lectura actividades adicionales que permita que realmente se comprenda lo que se haya leído. Finalmente se concluye que, para el logro de la comprensión lectora como una competencia, ésta tiene que adquirir conocimientos, desarrollar procesos cognitivos y actitudinales y desarrollar habilidades lingüísticas (Cassany, Luna \& Sanz, 2005).

Ausubel (1963) citado por Solé (2004) realiza una explicación de aprendizaje significativo desde un enfoque constructivista:

Aprender algo equivale a formarse una representación, un modelo propio, de aquello que se presenta como un objeto de aprendizaje, implica poder atribuirle significado al contenido en cuestión, en un proceso que conduce a una construcción personal, subjetiva, de algo que existe objetivamente. Ese proceso remite a la posibilidad de relacionar de una forma no arbitraria y sustantiva de lo que ya se sabe y lo que se pretende aprender. (p. 38).

Desde esta perspectiva existe un vínculo entre leer, comprender y aprender porque se lee para aprender de manera significativa, este proceso se realiza cuando realmente se comprende lo que se lee, esta afirmación es válida cuando la lectura informa al lector las nuevas perspectivas y conocimientos se acerca al mundo de significados preexistentes en el lector. Para ello se utiliza una serie de estrategias para asegurar el aprendizaje y lograr la meta comprensión, las mismas que parten del uso de estrategias de lectura: antes, durante y después de la lectura; lectura lenta, relectura, autopreguntas sobre el contenido de la lectura, uso del subrrayado, sumillado, elaboración de resúmenes, uso de organizadores de conocimientos entre otros.

Vallés (2005) considera que un estudiante que sea competente en comprender lo que lee tendrá acceso a la cultura y al aprendizaje en todos los ámbitos, competencia que además es básica en la búsqueda y localización de la información, así como para resolver problemas cotidianos y académicos y lo más importante le permitirá desarrollar su imaginación. Y a nivel cognitivo propiciará al estudiante desarrollar sus capacidades y funciones de procesamiento de la información para valorarla e interiorizarla.

Con respecto a los componentes o dimensiones considerados para la variable comprensión lectora, se tomaron en cuenta los niveles propuestos por Solé (1987):

Comprensión lectora literal, es el más elemental, donde el lector solo hace un reconocimiento sintáctico y semántico de la lectura, solo sin ninguna intervención de la estructura cognitiva ni intelectual, en este nivel solo se hace un reconocimiento de la información que es explícita como las ideas principales y secundarias, la secuencia de acciones, identificación de lugares, actores, hechos y la identificación de razones de los sucesos (Gordillo \& Flórez, 2009). 
Comprensión lectora inferencial, en la que juegan un papel importante los conocimientos previos almacenados en la memoria a largo plazo que tenga el estudiante, porque ello va a permitir que construya los significados del texto leído Vega (2012) esta construcción se da a través de tres procesos cognitivos: inferir, resumir y elaborar el significado del texto buscando en su red de relaciones y asociaciones, para explicar el texto más ampliamente porque le agrega más información, las experiencias previas, formula hipótesis, agrega nuevas ideas además que requiere mayor nivel de abstracción.

Comprensión lectora valorativa, considerado el nivel más alto ya para llegar a este, el lector debe haber desarrollado la capacidad de emitir juicios de valor de la lectura realizada, lo que le permitirá con argumentos aceptar o rechazar la propuesta luego de haber sido evaluado. Los criterios de juicio que puede utilizar el lector tienen el carácter de exactitud, aceptabilidad o de probabilidad (Gordillo \& Flórez, 2009).

\section{Método}

La investigación se trabajó bajo el enfoque cuantitativo y es de tipo sustantiva, el diseño considerado fue el correlacional causal, ya que se buscó hallar relaciones de tipo causa - efecto entre las variables estrategias de aprendizaje autónomo y la comprensión lectora.

Los instrumentos utilizados para recolectar la información fueron el Cuestionario de estrategias de trabajo autónomo (CETA) de la Universidad de León - España, con 45 ítems distribuidos en seis tipos de estrategias: estrategias de ampliación, de colaboración, de conceptualización, planificación, preparación de exámenes y participación, cuestionario que presenta un diseño tipo Likert con una escala ordinal de 1 al 5 desde nunca hasta siempre. El resultado del análisis factorial del Cuestionario de estrategias de trabajo autónomo (CETA) arrojó en su versión original un índice Kaiser - Meyer - Olkin (KMO) de 0.845, adicionalmente la prueba de esfericidad de Barlett tiene una significatividad de .000 que implica que existe interrelaciones significativas entre las variables que informan la adecuación de los datos a un modelo de análisis factorial.

En cuanto la comprensión lectora se construyó la prueba PECL 2 para medir niveles de comprensión de la lectura: literal, inferencial y criterial, elaborándose una rúbrica para evaluar el desempeño de los estudiantes, el instrumento pasó por la validez de contenido, a cargo de expertos en el tema, dando una opinión final de aplicable; así mismo la confiabilidad arrojó un Alfa de 0.657, considerada para pruebas de rendimiento como aceptable. Ambos instrumentos fueron aplicados al total de estudiantes del 5to de secundaria de tres Instituciones Educativas Públicas, una de ellas ubicada en Majes, Arequipa con 51 estudiantes y dos en Villa Rica, Pasco, con 93 estudiantes 


\section{Resultados}

\section{Tabla 1.}

Estrategias de Aprendizaje Autónomo de los estudiantes del 5to grado de secundaria de IE públicas de Pasco y Arequipa, expresado en niveles.

\begin{tabular}{lccccccc}
\hline Niveles & $\begin{array}{c}\text { Aprend } \\
\text { izaje } \\
\text { autóno } \\
\text { mo }\end{array}$ & $\begin{array}{c}\text { Estrategi } \\
\text { as de } \\
\text { ampliaci } \\
\text { ón }\end{array}$ & $\begin{array}{c}\text { Estrategias } \\
\text { de } \\
\text { colaboraci } \\
\text { ón }\end{array}$ & $\begin{array}{c}\text { Estrategia } \\
\text { s de } \\
\text { conceptua } \\
1\end{array}$ & $\begin{array}{c}\text { Estrategi } \\
\text { as de } \\
\text { planifica } \\
\text { ción }\end{array}$ & $\begin{array}{c}\text { Estrategi } \\
\text { as de } \\
\text { Prep. } \\
\text { Exámene } \\
\text { s }\end{array}$ & $\begin{array}{c}\text { Estrategias } \\
\text { de } \\
\text { participaci } \\
\text { ón }\end{array}$ \\
\hline En inicio & 16.0 & 15.3 & 23.3 & 20.7 & 60.0 & 6.7 & 12.0 \\
En proceso & 79.3 & 74.0 & 74.0 & 79.3 & 40.0 & 60.7 & 74.0 \\
Logro & 4.7 & 10.7 & 2.7 & 0.0 & 0.0 & 32.7 & 14.0 \\
& 100. & 100.0 & 100.0 & 100.0 & 100.0 & 100.0 & 100.0 \\
Total & 0 & & & & & & \\
\hline
\end{tabular}

Nota: Valores expresados en porcentaje

La tabla muestra el nivel de uso de las estrategias de aprendizaje autónomo, así como de las seis dimensiones correspondientes de los estudiantes del 5to grado de secundaria de IE públicas de Pasco y Arequipa. El 79.3\% de los estudiantes se encuentran en proceso con respecto al uso de estrategias de aprendizaje autónomo y solo un 4.7\% alcanzó el nivel logrado. En cuanto a las dimensiones, las estrategias de planificación fueron las que evidenciaron mayores dificultades, con un $60 \%$ de estudiantes en el nivel inicio, mientras que las que obtuvieron mejores resultados fueron las estrategias de preparación con un $32.7 \%$ de estudiantes en nivel logrado.

Tabla 2.

Nivel de comprensión lectora de los estudiantes del 5to grado de secundaria de IE públicas de Pasco y Arequipa

\begin{tabular}{lcccc}
\hline Niveles & $\begin{array}{c}\text { Comprensió } \\
\text { n lectora }\end{array}$ & Nivel literal & $\begin{array}{c}\text { Nivel } \\
\text { inferencial }\end{array}$ & Nivel valorativo \\
\hline En inicio & 26.7 & 38.7 & 17.3 & 68.7 \\
En proceso & 62.7 & 59.3 & 52.0 & 26.7 \\
Logro & 10.7 & 2.0 & 30.7 & 4.7 \\
Total & 100.0 & 100.0 & 100.0 & 100.0 \\
\hline
\end{tabular}

Nota: Valores expresados en porcentaje

Los resultados que muestran la tabla 2 confirman resultados ya conocidos como país, solo un $10.7 \%$ alcanzó el nivel logrado, $62.7 \%$ en proceso de logro y $26.7 \%$ en nivel de inicio. En 
cuanto a las dimensiones de comprensión lectora, el nivel valorativo presentó mayores dificultades con un $68,7 \%$ de estudiantes en inicio frente a un $30.7 \%$ de estudiantes que en el nivel inferencial resultaron con logrado.

Tabla 3.

Ajuste del modelo

\begin{tabular}{|c|c|c|c|c|c|}
\hline & Modelo & $\begin{array}{c}-2 \log \text { de la } \\
\text { verosimilitu } \\
\mathrm{d}\end{array}$ & $\begin{array}{l}\text { Chi- } \\
\text { cuadrado }\end{array}$ & $\mathrm{gl}$ & Sig. \\
\hline & $\begin{array}{l}\text { Sólo } \\
\text { intersección }\end{array}$ & 152.973 & & & \\
\hline $\begin{array}{l}\text { Dimensiones de la variable } \\
\text { Aprendizaje autónomo }\end{array}$ & Final & 102.545 & 50.428 & 10 & .000 \\
\hline $\begin{array}{l}\text { Indicadores de la dimensión } \\
\text { Ampliación }\end{array}$ & & $\begin{array}{l}75.345 \\
39.166\end{array}$ & 36.178 & 4 & .000 \\
\hline $\begin{array}{l}\text { Indicadores de la dimensión } \\
\text { Colaboración }\end{array}$ & & $\begin{array}{l}54.870 \\
36.001\end{array}$ & 18.870 & 4 & .001 \\
\hline $\begin{array}{l}\text { Indicadores de la dimensión } \\
\text { Conceptualización }\end{array}$ & & $\begin{array}{l}72.157 \\
31.311\end{array}$ & 40.845 & 4 & .000 \\
\hline $\begin{array}{l}\text { Indicadores de la dimensión } \\
\text { Planificación }\end{array}$ & & $\begin{array}{l}58.796 \\
32.250\end{array}$ & 26.546 & 4 & .000 \\
\hline $\begin{array}{l}\text { Indicadores de la dimensión } \\
\text { Preparación de exámenes }\end{array}$ & & $\begin{array}{l}58.122 \\
35.168\end{array}$ & 22,955 & 4 & .000 \\
\hline $\begin{array}{l}\text { Indicadores de la dimensión } \\
\text { Participación }\end{array}$ & & 66.926 & 26.390 & 4 & .000 \\
\hline Función de vínculo: Logit. & & & & & \\
\hline
\end{tabular}

La tabla 3 muestra que en todos los casos el valor de significación obtenido es menor al nivel de significación propuesto de 0,05 por lo que se afirma que el modelo no es adecuado solo con la constante y requiere de la variable propuesta con sus dimensiones e indicadores. 
Tabla 4.

Pseudo R-cuadrado

\begin{tabular}{lccccccc}
\hline & $\begin{array}{l}\text { Aprendizaj } \\
\mathrm{e} \\
\text { autónomo }\end{array}$ & $\begin{array}{c}\text { Estrategias } \\
\text { de } \\
\text { ampliación }\end{array}$ & $\begin{array}{c}\text { Estrategias } \\
\text { de } \\
\text { colaboració } \\
\mathrm{n}\end{array}$ & $\begin{array}{c}\text { Estrategias } \\
\text { de } \\
\text { conceptual }\end{array}$ & $\begin{array}{c}\text { Estrategias } \\
\text { de } \\
\text { planificaci } \\
\text { on }\end{array}$ & $\begin{array}{c}\text { Estrategias } \\
\text { de Prep. } \\
\text { Exámenes }\end{array}$ & $\begin{array}{c}\text { Estrategias } \\
\text { de } \\
\text { participación }\end{array}$ \\
\hline $\begin{array}{l}\text { Cox y } \\
\text { Snell }\end{array}$ & .286 & .214 & .118 & .238 & .162 & .142 & .161 \\
Nagelkerke & .344 & .258 & .143 & .287 & .196 & .171 & .195 \\
McFadden & .190 & .136 & .071 & .154 & .100 & .087 & .100 \\
\hline
\end{tabular}

Función de vínculo: Logit.

La tabla 4 muestra los resultados obtenidos de la prueba de Nagelkerke que explica la variabilidad de la comprensión lectora explicada por el modelo, tanto por las dimensiones como por los indicadores de cada dimensión propuesta.

Se aprecia que el $34.4 \%$ de la variable comprensión lectora es explicada por el uso de estrategias de aprendizaje autónomo. Así mismo de ese 34\%, la comprensión lectora es explicada en un $25.8 \%$ por las estrategias de ampliación, $14.3 \%$ por las estrategias de colaboración, $28.7 \%$ por las estrategias de conceptualización, $19.6 \%$ por las estrategias de preparación de exámenes y finalmente un $19.5 \%$ por las estrategias de participación.

Tabla 5.

Estimación de parámetros

\begin{tabular}{|c|c|c|c|c|c|c|c|c|}
\hline & & $\begin{array}{l}\text { Estima } \\
\text { ción }\end{array}$ & $\begin{array}{l}\text { Error } \\
\text { típ. }\end{array}$ & Wald & gl & Sig. & Intervalo $\mathrm{d}$ & onfianza \\
\hline & & & & & & & $\begin{array}{l}\text { Límite } \\
\text { inferior }\end{array}$ & $\begin{array}{l}\text { Límite } \\
\text { superior }\end{array}$ \\
\hline E. A.A. & [amplia=1] & $\begin{array}{c}- \\
2.444\end{array}$ & .894 & 7.478 & 1 & .006 & -4.195 & -.692 \\
\hline E. Amp & $\begin{array}{l}\text { [actcomple } \\
\text { ment }=1]\end{array}$ & -2.734 & .837 & $\begin{array}{r}10.66 \\
0\end{array}$ & 1 & .001 & -4.376 & -1.093 \\
\hline E. Concp & [visual=1] & -2.648 & .753 & $\begin{array}{r}12.38 \\
2\end{array}$ & 1 & .000 & -4.124 & -1.173 \\
\hline E. exam & {$[$ lectura $=2]$} & 1.426 & .454 & 9.861 & 1 & .002 & -2.316 & -.536 \\
\hline E. Part. & {$[\mathrm{duda}=1]$} & -2.228 & .661 & $\begin{array}{r}11.35 \\
7\end{array}$ & 1 & .001 & -3.525 & -.932 \\
\hline
\end{tabular}

La tabla 5 muestra el resumen de los indicadores y niveles que más inciden en la variable comprensión lectora, donde se observa que no todos los indicadores tienen carácter explicativo 
en el modelo, solo algunos de las dimensiones estrategias de ampliación, conceptualización, preparación para los exámenes y participación. Con respecto al indicador predominante de las estrategias de ampliación son las actividades complementarias las de mayor carácter explicativo, en cuanto a la dimensión estrategias de conceptualización es el uso de esquemas visuales el indicador predominante, con respecto a las estrategias de preparación para los exámenes es la lectura de materiales; finalmente para la dimensión estrategias de participación el indicador predominante que incide en la comprensión lectora es la aclaración de dudas.

\section{Discusión}

En la población donde se realizó el estudio, las estrategias de aprendizaje autónomo se relacionan con la comprensión lectora, cada una de las dimensiones tienen carácter explicativo en mayor o menor medida, resultados similares fueron los obtenidos por los iraníes Koosha, Abdollahi y Karimi (2016), quienes hallaron una correlación alta entre el aprendizaje autónomo (AA) y comprensión lectora (CL) y una explicación del $82 \%$ del AA sobre la CL, también se encuentran los obtenidos por Zarei y Gahremani en el 2010 quienes en universitarios del mismo país, hallaron relación baja, entre el AA y la CL, no obstante en el análisis de regresión no se encontró ningún tipo de predicción. Existen pocos estudios de corte empírico relacionados directamente con el objetivo de este trabajo que puedan servir como referente a nivel nacional, uno de los pocos es el estudio de Sevillano (2015), quien halló relación entre las estrategias de aprendizaje, la motivación y la CL. Al respecto López- Aguado (2010) sostiene que al utilizar estrategias de aprendizaje independiente, el estudiante regulará y controlará mejor su aprendizaje y en el proceso lector este aspecto es de vital importancia, cuando no hay la compañía del docente o un mediador del proceso lector, es el individuo quien debe emplear diversas estrategias para asegurar que realmente exista una lectura efectiva, por ello es importante que conozca qué hacer y cómo asegurar este proceso, llamado metacomprensión (Ríos 1991), es decir el conocimiento y regulación de diversas estrategias y recursos de corte cognitivo que permiten enfrentarse a un texto. Así las estrategias de ampliación, conceptualización, preparación y participación donde se hallaron indicadores que explican la CL de manera significativa, implican que si un estudiante tiene estrategias para ampliar la información y mejorar el entendimiento de algún concepto, sabe y pone en práctica como realizar organizadores visuales, se planifica en cuanto al uso del tiempo, autoevalúa su aprendizaje y participa en aula de forma activa, tendrá según el modelo hallado, una comprensión exitosa del texto.

\section{Referencias}

Ceplan (2014) La educación del futuro u el futuro de la educación, Serie: Avance de investigación $\mathrm{N}^{\mathrm{o}}$ 2. Lima: Punto y grafía.

Ceplan (2017) INEI: Sistema de Monitoreo y Seguimiento de los Indicadores de los Objetivos de Desarrollo Sostenible por Aníbal Sánchez 19.12.16 Recuperado de: https://www.ceplan.gob.pe/documentos_/sistema-de-monitoreo-y-seguimiento-de-losindicadores-de-los-objetivos-de-desarrollo-sostenible/

El Comercio (2016, 06 de diciembre). Perú sale del último lugar en la prueba PISA 2015 Recuperado de: https://elcomercio.pe/peru/peru-sale-lugar-prueba-pisa-2015-152124

Esteban, M., Ruiz, C., \&Cerezo, F. (1996) Los estilos de aprendizaje y el rendimiento en Ciencias Sociales y en Ciencias de la Naturaleza en estudiantes de Secundaria. Anales de Psicología, 12(2), 153-166. Recuperado de: http://www.um.es/analesps/v12/v12_2/0412-2.pdf 
Gaeta, M. (2015). Aspectos personales que favorecen la autorregulación del aprendizaje en la comprensión de textos académicos en estudiantes universitarios. REDU Revista de docencia universitaria 13(2), 17-35. Doi: https://doi.org/10.4995/redu.2015.5436

Gordillo, A., \& Flórez, M. (2009). Los niveles de comprensión lectora: hacia una enunciación investigativa y reflexiva para mejorar la comprensión lectora en estudiantes universitarios. Revista de actualidad pedagógica, 63, Doi: https://doi.org/10.19052/ap.1048

Guevara, M. (2014). Estrategias de Aprendizaje Cooperativo y Comprensión Lectora con textos filosóficos en Estudiantes de Filosofía de la Facultad de Educación de la Universidad Nacional Amazónica de Madre de Dios, año 2012 (Tesis de Maestría). Universidad Nacional Mayor de San Marcos, Lima. Recuperado de: http://cybertesis.unmsm.edu.pe/handle/cybertesis/3957

Koosha, M., Abdollahi, A., \& Karimi, F. (2016). The Relationship among EFL Learners' Selfesteem, Autonomy, and Reading Comprehension. Theory and Practice in Language Studies, 6 (1), 68-78. Doi: http://dx.doi.org/10.17507/tpls.0601.09

Lòpez, A. (22 de febrero, 2002) Los expertos relacionan la falta de estrategias de aprendizaje y el fracaso. Diario El País, Madrid. Recuperado de: https://elpais.com/diario/2002/02/25/educacion/1014591601_850215.html

López-Aguado, M. (2010). Diseño y análisis del Cuestionario de estrategias de trabajo autónomo (CETA) para estudiantes universitarios Revista de Psicodidáctica, 15(1), 77-99. Recuperado de: http://www.ehu.eus/ojs/index.php/psicodidactica/article/view/735

Mayor, J., Suengas, A., \& Gonzalez, J. (1993). Estrategias metacognitivas. Aprender a aprender y aprender a pensar. Madrid. Síntesis.

Pinzás, J. (2006). Guía de estrategias metacognitivas para desarrollar la comprensión lectora. Lima. Ministerio de Educación.

Ríos, P. (1991). Metacognición y comprensión de la lectura. En A. Puente (Comp.). Comprensión de la lectura y acción docente. Madrid: Pirámide.

Sevillano, M. (2015). Relación entre las estrategias de aprendizaje, la motivación y la comprensión lectora en los estudiantes de cuarto año de Secundaria de una Institución Educativa Particular del Distrito de Chorrillos (Tesis de Maestría). Universidad Ricardo Palma. Lima. Recuperado de: http://repositorio.urp.edu.pe/handle/urp/733

Solé, I. (1987). Las posibilidades de un modelo teórico para la enseñanza de la comprensión lectora. Infancia y Aprendizaje, 10, 39-40. Doi: https://doi.org/10.1080/02103702.1987.10822170

Solé, I. (2004). Estrategias de lectura (15a ed.). Barcelona: Grao

Solé, I. (2007). La lectura como transformación del pensamiento. Proyecto de Lectura para Centros Escolares (plec). Madrid: Fundación Germán Sánchez Ruipérez

Solórzano, J., \& Montero, E. (2011). Construcción y validación de una prueba de comprensión de lectura mediante el modelo de Rasch. Actualidades investigativas en educación, 11(2), 1-27. Doi: https://doi.org/10.15517/AIE.V11I2.10188

Vallés, A. (2005) Comprensión lectora y procesos psicológicos. Liberabit, 11(11), 49-65. Recuperado de: http://pepsic.bvsalud.org/pdf/liberabit/v11n11/v11n11a07.pdf

Vega, C. (2012). Niveles de comprensión lectora en alumnos de quinto grado de primaria de una Institución educativa de Bellavista Callao (Tesis de Maestría). Universidad San Ignacio de Loyola, Lima.

Zarei, A.A., \& Gahremani, K. (2010). On the relationship between learner autonomy and reading comprehension. Tell, 3 (10), 81-99. Recuperado de: http://www.ikiu.ac.ir/publicfiles/profiles/items/090ad_1424769045.pdf 